Genetic Epidemiology 19:178-190 (2000)

\title{
Twin Study of Adolescent Genetic Susceptibility to Mosquito Bites Using Ordinal and Comparative Rating Data
}

\author{
Katherine M. Kirk, ${ }^{1 *}$ Lindon J. Eaves, ${ }^{2}$ Joanne M. Meyer, ${ }^{3}$ Allan Saul, ${ }^{4}$ \\ and Nicholas G. Martin ${ }^{1}$ \\ ${ }^{1}$ The Queensland Institute of Medical Research and Joint Genetics Program, \\ The University of Queensland, Brisbane, Queensland, Australia \\ ${ }^{2}$ Virginia Institute of Psychiatric and Behavioral Genetics, Richmond, Virginia \\ ${ }^{3}$ Millenium Pharmaceuticals, Inc., Boston, Massachusetts \\ ${ }^{4}$ Australian Centre for International and Tropical Health and Nutrition, University \\ of Queensland, Brisbane, Queensland, Australia
}

\begin{abstract}
Ordinal and comparative rating measures of mosquito attraction and mosquito bite frequency and symptoms were administered in a self-report questionnaire format to a sample of 197 monozygotic and 326 dizygotic Australian adolescent twin pairs at age 12 between 1992 and 1999, in order to investigate the environmental and possibly genetic determinants of variation between individuals. Repeat measures were obtained from the twin pairs at age 14. Ordinal variable measures, although providing some support for genetic effects on mosquito susceptibility, were affected by low repeatability. However, analysis of a comparative rating variable "compared with your twin, who is bitten by mosquitoes more often?" indicated a strong genetic influence on frequency of being bitten by mosquitoes, with no significant differences observed between males and females. Comparative rating questionnaire items are a potentially valuable tool for complementing and improving the results obtained from more conventional absolute measures. Genet. Epidemiol. 19:178-190, 2000. () 2000 Wiley-Liss, Inc.
\end{abstract}

Key words: environment; genetics; insect bites; mosquito; twins

\footnotetext{
Contract grant sponsor: Queensland Cancer Fund; Contract grant sponsor: National Health and Medical Research Council; Contract grant number: 950998.

*Correspondence to: Dr. K. M. Kirk, Queensland Institute of Medical Research, Post Office, Royal Brisbane Hospital, Brisbane QLD 4029 Australia. E-mail: kathE@qimr.edu.au
}

Received 24 March 1999; Accepted 20 September 1999

(C) 2000 Wiley-Liss, Inc. 


\section{INTRODUCTION}

The degree of attraction of mosquitoes has been demonstrated to vary considerably between individual humans. Many studies have been performed to determine the causes of this variation, with factors such as age [Maibach et al., 1966], warmth and moisture [Brown, 1966; Wright, 1975], carbon dioxide $\left(\mathrm{CO}_{2}\right)$ emission [Brown, 1966; Snow, 1970; Gillies, 1980], and human body odour [Brouwer, 1960; see also Knols and De Jong, 1996], all found to affect mosquito host-seeking behavior. Investigation of these effects is an important part of mosquito control research, aimed at not only reducing the nuisance of mosquitoes but also the incidence of mosquitoborne diseases such as malaria, dengue haemorrhagic fever, and several forms of encephalitis [Fradin, 1998].

Symptoms of mosquito bites include immediate pruritic weals and delayed bite papules, with immediate reactions believed to be mediated by IgE and histamine, and cellmediated immunity responsible for delayed reactions [Fradin, 1998]. As with attractiveness to mosquitoes, cutaneous responses to bites also differ between individuals, with respect to both onset time and severity of reaction. This has been attributed to a sensitization/ desensitization process [Mellanby, 1946; McKiel and West, 1961].

The perception of being bitten by mosquitoes is dependent on several factors, two of which are the actual frequency of being bitten (which in turn could be reasonably expected to depend on an individual's degree of mosquito attraction), and the severity of reaction to a mosquito bite. People with no reaction to mosquito bites are far less likely to realise mosquito bites have occurred than those who have severe

cutaneous responses. The lower than expected correlation observed between reported bites and mosquito trap counts [Read et al., 1994] can be considered as being at least partly due to the combined action of these factors.

Previous studies of the attraction of mosquitoes to humans have focussed on environmental factors, and little or no research exploring the possibility of genetic influences appears to have been published. The aim of this paper is to analyse ordinal and comparative rating data to investigate the environmental and possibly genetic determinants of variation in mosquito attraction and bite reactions between individuals, using an Australian adolescent twin sample.

\section{MATERIALS AND METHODS}

\section{Sample}

This study was conducted in the context of an ongoing longitudinal investigation of melanocytic naevus (mole) development in adolescent twins in Queensland, Australia, the sample and clinical protocol of which have been described elsewhere [Aitken et al., 1994]. At age 12 and again at age 14, participating twins and their mothers were invited to visit the Queensland Institute of Medical Research, where amongst other procedures questionnaires were completed individually by the twins under supervised conditions and without opportunity to confer regarding responses. Zygosity of twins was initially established by the physical appearance of the twins and mother's report. In the absence of complete certainty, genotyping was undertaken using eight independent polymorphic markers and three blood type indicators.

The present analysis involves the 197 monozygotic (98 female and 99 male) 
and 326 dizygotic ( 83 female, 88 male, and 155 unlike-sex) twin pairs who first visited the Institute between May 1992 and August 1999, with a mean age of 12.2 (standard deviation, 0.37) years. As of August 1999, 380 of these twin pairs had also completed their second visit, with a mean age at visit 2 of 14.1 (standard deviation, 0.20 ) years. The average time interval between the two visits was 1.8 (standard deviation, 0.35 ) years. As a method of validating the melanocytic naevi counting techniques used in the general study from which this data is obtained, 33 pairs of twins were additionally re-examined at least 2 weeks following their first visit (range 2-11 weeks, median 4 weeks).

The dominant mosquito species in the Brisbane area vary both spatially and with time. In the eastern coastal suburbs, the salt water breeding mosquito Aedes vigilax can occur in very large numbers, depending on tides and weather. Further inland, Culex annulirostris and the peridomestic Aedes notoscriptus are relatively more important, with locally high numbers of Aedes funereus occurring in some suburbs [Ritchie et al., 1997]. While all these mosquitoes are attracted to relatively nonspecific attractants such as $\mathrm{CO}_{2}$, they show different responses to the specific attractant, 1-octen-3-ol, which is believed to be one of the odours preferentially associated with attraction to mammals.

\section{Measures}

Included in the questionnaire were four ordinal items and one comparative item pertaining to mosquitoes. Items 1 and 2 were "At an outdoor evening activity in summer (like a barbecue) would you be annoyed by mosquito bites" and "At an outdoor activity in summer (like a barbecue) would you use a mosquito repellent like Rid or Aerogard," respectively. The third question ("If you are bitten by a mosquito how long does the bite usually remain itchy?") and fourth question ("How would you usually describe a bite?") both relate to bite symptoms. Response sets for these items are listed in Table III. Item five asked twins "Compared with your twin, who is bitten by mosquitoes more often?", with possible responses of "me," "my twin," or "same."

\section{Statistical Methods}

Correlations between variables are calculated on the assumption that underlying each variable is a continuum of liability that is normally distributed in the population. Ordinal variables arise when thresholds, often arbitrary, are imposed on this liability continuum. PRELIS 2.20 [Jöreskog and Sörbom, 1998] has been used to estimate polychoric correlations between ordinal variables, and Mx [Neale, 1997] was used for equivalence testing of imposed thresholds and correlations. Multivariate analysis was conducted using LISREL 8.20 [Jöreskog and Sörbom, 1998]. Other statistical tests were performed using PROC FREQ in SAS 6.11 [SAS Institute, 1996].

Analysis of the comparative rating data was achieved using the model for comparative ratings of within-family differences devised by Eaves et al. [1991], which uses comparative information from two raters to estimate the reliable within-family variance of differential experience. True individual differences between pairs are unknown; however, it is assumed that these latent intrapair differences $D$ have a distribution $N\left[0, \sigma_{\mathrm{w}}^{2}\right]$, where $\sigma_{\mathrm{w}}^{2}$ is the within-pair variance for the latent attributes. In the 
case of $m$ possible response categories, the log-likelihood of the overall set of possible pairwise comparisons is

$$
L L\left(\sigma_{w}^{2}, T\right)=\sum_{i=1}^{m} \sum_{j=1}^{m} N_{i j} \ln P_{i j}+C,
$$

where the $N_{i j}$ are the observed contingency table cell frequencies, and the $P_{i j}$ are the joint probabilities of raters 1 and 2 endorsing categories bounded by thresholds $T_{i}$ and $T_{i+1}$, and $T_{j}$ and $\mathrm{T}_{j+1}$, respectively

$$
P_{i j}=\int_{-\infty}^{\infty} \phi(D)\left[\int_{T_{i}-D}^{T_{i+1}-D} \phi\left(x_{1} \mid D\right) d x \int_{T_{j}+D}^{T_{j+1}+D} \phi\left(x_{2} \mid D\right) d x\right] d D .
$$

The log-likelihood can then be maximised with respect to threshold values and the within-family variance.

Joint analysis of comparative ratings on different kinds of relatives (in this case, monozygotic and dizygotic twins) can be used to determine whether perceived differences between twins reflect responses to genetic or non-genetic factors. With genetically informative data, the within-family variance may be parameterised in terms of genetic and environmental components, with respect to which the log likelihood is maximised. For MZ twins, the within-family variance is totally due to environmental factors, since they are genetically identical: $\sigma_{\mathrm{w}}^{2}=\sigma_{\mathrm{e}}^{2}$. However, dizygotic twins only share on average $50 \%$ of their genes, so that the within-family variance has a genetic component: $\sigma_{\mathrm{w}}^{2}=\sigma_{\mathrm{e}}^{2}$ $+0.5 \sigma_{\mathrm{g}}^{2}$. Submodels of the full comparative model can be fitted to the data, and compared to the full model using likelihood ratio chi-square tests.

Where information is available from males and females in MZ twin pairs and DZ same-sex and opposite-sex twin pairs, modelling techniques can be extended to include considerations of whether there are sex differences in gene expression. Scalar sex-limitation occurs when the same genes affect both males and females, with the magnitude of the effect differing only by some constant multiple over all the loci involved [Neale and Cardon, 1992] and the expected genetic correlation between opposite-sex twin pairs remaining at 0.5 . This expectation is no longer true in the case of non-scalar sex-limitation, where quite different genes may control the expression of a trait in the two sexes. In this case, the correlation between the genes affecting a trait in males and females, $r_{a_{F M}}$, would be significantly less than one and the expected genetic correlation of DZ opposite-sex co-twins would be less than 0.5. A summary of the within-pair differences expected for monozygotic and dizygotic twin pairs appears in Table I. The phenomenon of sex limitation in the expression of genetic variance is quite distinct from the question of sex differences in the mean, which can be estimated from the contingency table for DZ opposite sex twins as a significant difference between the number of female twins and male twins reliably reported as being more affected than their co-twins.

It is important to note that the estimated within-family variance components are components of "reliable" within-family differences (those about which twins agree). Disagreement between twins introduces error variance and is accounted for by allowing a finite chance of endorsing any response category given a true difference between family members. 
TABLE I. Expected Within-Pair Variances for Monozygotic Male (MZM) and Female (MZF) and Dizygotic Same-Sex Male (DZM), Same-Sex Female (DZF), and Opposite-Sex (DZFM) Twin Pairs

\begin{tabular}{ll}
\hline Twin pair type & \multicolumn{1}{c}{ Expected within-pair variance } \\
\hline MZM & $\sigma_{e_{m}}^{2}$ \\
MZF & $\sigma_{e_{f}}^{2}$ \\
DZM & $\sigma_{e_{m}}^{2}+\frac{1}{2} \sigma_{g_{m}}^{2}$ \\
& $\sigma_{e_{f}}^{2}+\frac{1}{2} \sigma_{g_{f}}^{2}$ \\
DZF & $\frac{1}{2}\left(\sigma_{e_{m}}^{2}+\sigma_{e_{f}}^{2}+\sigma_{g_{m}}^{2}+\sigma_{g_{f}}^{2}-2 \times \frac{1}{2} \times r_{a_{F M}} \sigma_{g_{m}} \sigma_{g_{f}}\right)$ \\
DZFM & $=\frac{1}{2}\left(\sigma_{e_{m}}^{2}+\sigma_{e_{f}}^{2}+\sigma_{g_{m}}^{2}+\sigma_{g_{f}}^{2}-r_{a_{F M}} \sigma_{g_{m}} \sigma_{g_{f}}\right)$ for non-scalar sex-limitation \\
& $=\frac{1}{2}\left(\sigma_{e_{m}}^{2}+\sigma_{e_{f}}^{2}+\sigma_{g_{m}}^{2}+\sigma_{g_{f}}^{2}-\sigma_{g_{m}} \sigma_{g_{f}}\right)$ for scalar sex-limitation \\
& $=\sigma_{e_{m}}^{2}+\frac{1}{2} \sigma_{g_{m}}^{2}=\sigma_{e_{f}}^{2}+\frac{1}{2} \sigma_{f}^{2}$ for no sex-limitation effect \\
&
\end{tabular}

\section{RESULTS}

\section{Test-Retest and Long-Term Stability Data}

Comparison of individuals' item responses collected at visit 1 with those collected at later times provides an indicator of the stability of the measures being used. From the 33 pairs of individuals re-examined 2-11 weeks after visit 1, complete data for the items of interest in this study were available for 48 individuals. As the correlations in Table II indicate, the variable relating to being annoyed by mosquitoes is highly subject to even short-term variability, while the other items appear to have reasonable stability. The repeatability over the 2-year period between visit 1 and visit 2 is lower again. Values for the comparative rating data were obtained using the comparative rating technique described above, substituting data obtained for individuals at the two time-points for "twin 1" and "twin 2" data. As a result, the estimate obtained is the proportion of the "withinindividual" variance that is reliable.

TABLE II. Test-Retest Correlations for Ordinal and Comparative Ratings of Mosquito Bite Effects on Adolescent Australian Twins Aged 12, With Repeat Measurements at 2-11 Weeks $(n=48) *$

\begin{tabular}{lcc}
\hline Variable & Time interval $=2-11$ weeks & Time interval = 2 years \\
\hline Annoyed by mosquitoes & $0.56 \pm 0.11$ & $0.28 \pm 0.04$ \\
Use mosquito repellent & $0.70 \pm 0.08$ & $0.44 \pm 0.04$ \\
Duration of bite itchiness & $0.73 \pm 0.07$ & $0.57 \pm 0.03$ \\
Bite description & $0.77 \pm 0.07$ & $0.50 \pm 0.04$ \\
Bite frequency, twin comparative rating & 0.81 & 0.67 \\
\hline
\end{tabular}

Also shown are correlations between original ratings and those recorded at age $14(n=759)$. Values for the comparative rating variable are the proportions of the "within-twin" variance that are reliable in each case. 


\section{Ordinal Variables}

Response frequencies for the ordinal variables (mosquito annoyance, repellent use, duration of itchiness, and bite description) appear in Table III. No significant differences in responses arising from seasonal factors (the time of year in which the twins were interviewed) or mosquito population (as indicated by residential location) were detected. Testing of response category thresholds using Mx found significant differences between males and females for use of mosquito repellent and description of mosquito bites at visit $1(P=0.008 ; P=0.01)$ and visit $2(P=0.001 ; P$ $<0.001)$, and duration of bite itchiness for visit 2 only $(P=0.003)$. No significant change in response frequencies was observed for males or females for any items in the 2-year period between measurements.

The twin-pair correlations observed for the various zygosity groups were then tested for equivalence, with the results for visit 1 appearing in Table IV. No significant difference between the correlation values for males and females was observed ( $P>0.2$ for all variables), and a comparison of models 1 and 2 indicates that the twin-pair correlations for monozygotic and dizygotic twins can be equated, providing no evidence for any significant genetic influence on these variables. Similar results were obtained for the data collected at visit 2 (age 14). Equating all twin-pair correlations to zero (model 3 ) provides a significantly poorer fit for all variables except being annoyed by mosquitoes at age 12, indicating the presence of significant familial aggregation for these variables. That is, genetic and/or common environment effects are present, but cannot be disentangled from each other using univariate

TABLE III. Response Frequencies (Percentages) for Ordinal Questionnaire Items on Mosquito Bite Effects on Australian Adolescents at Visit 1 (Age 12) and Visit 2 (Age 14)*

\begin{tabular}{lccccc} 
& \multicolumn{2}{c}{ Visit 1 $(\sim$ age 12) } & & \multicolumn{2}{c}{ Visit 2 ( age 14) } \\
\cline { 2 - 3 } \cline { 5 - 6 } & Females & Males & & Females & Males \\
\hline Annoyed by mosquitoes & $(n=517)$ & $(n=529)$ & & $(n=387)$ & $(n=373)$ \\
Never & 3.1 & 6.4 & & 3.6 & 8.3 \\
Sometimes & 43.7 & 44.0 & & 49.7 & 51.2 \\
Most times & 30.4 & 28.7 & & 30.1 & 27.3 \\
Always & 22.8 & 20.8 & & 16.6 & 13.1 \\
Use mosquito repellent & $(n=515)$ & $(n=528)$ & & $(n=386)$ & $(n=373)$ \\
Never & 8.0 & 13.4 & & 11.4 & 19.3 \\
Sometimes & 44.1 & 46.8 & & 48.7 & 50.1 \\
Most times & 28.9 & 24.1 & & 26.7 & 22.0 \\
Always & 19.0 & 15.7 & & 13.2 & 8.6 \\
Duration of bite itchiness & $(n=516)$ & $(n=525)$ & & $(n=386)$ & $(n=371)$ \\
Not at all & 5.0 & 9.9 & & 6.0 & 13.5 \\
A few minutes & 30.0 & 33.7 & & 26.9 & 35.0 \\
About half an hour & 25.2 & 21.5 & & 29.3 & 22.1 \\
An hour to a few hours & 14.5 & 12.6 & & 14.8 & 15.6 \\
A day or longer & 25.2 & 22.3 & & 23.1 & 13.7 \\
Bite description & $(n=516)$ & $(n=527)$ & & $(n=387)$ & $(n=373)$ \\
Can't see it & 4.3 & 9.1 & & 6.5 & 12.9 \\
A little red but no lump & 18.0 & 18.8 & & 12.7 & 18.5 \\
A small lump & 66.1 & 60.2 & 68.7 & 63.0 \\
A big lump & 11.6 & 12.0 & 12.1 & 5.6 \\
\hline
\end{tabular}

*Numbers of item responses vary slightly within visits due to missing values. 
TABLE IV. Table of Twin Pair Correlations for Ordinal Measures of Mosquito Bite Effect on Australian Adolescents Aged 12*

\begin{tabular}{|c|c|c|c|c|c|c|c|c|}
\hline \multirow[b]{2}{*}{ Model } & \multicolumn{2}{|c|}{ Annoyed by mosquitoes } & \multicolumn{2}{|c|}{ Use mosquito repellent } & \multicolumn{2}{|c|}{ Duration of bite itchiness } & \multicolumn{2}{|c|}{ Bite description } \\
\hline & $\mathrm{N}_{\text {pair }}$ & $r$ & $\mathrm{~N}_{\text {pair }}$ & $r$ & $\mathrm{~N}_{\text {pair }}$ & $r$ & $\mathrm{~N}_{\text {pair }}$ & $r$ \\
\hline $1 \mathrm{MZ}$ & 197 & 0.035 & 196 & 0.335 & 196 & 0.220 & 195 & 0.253 \\
\hline DZ & 326 & 0.034 & 324 & 0.196 & 322 & 0.196 & 325 & 0.140 \\
\hline \multirow[t]{2}{*}{2 All } & 523 & 0.035 & 520 & 0.243 & 518 & 0.205 & 520 & 0.183 \\
\hline & \multicolumn{2}{|c|}{$\Delta \chi_{1}^{2}<0.001 P \approx 1$} & \multicolumn{2}{|c|}{$\Delta \chi_{1}^{2}=1.976 P=0.160$} & \multicolumn{2}{|c|}{$\Delta \chi_{1}^{2}=0.061 P=0.805$} & \multicolumn{2}{|c|}{$\Delta \chi_{1}^{2}=1.085 P=0.298$} \\
\hline 3 All & $\begin{array}{l}\mathbf{5 2 3} \\
\Delta \chi_{1}^{2}\end{array}$ & $\begin{array}{r}\mathbf{0 . 0 0 0} \\
=0.504\end{array}$ & $\begin{array}{l}520 \\
\Delta \chi_{1}^{2}=\end{array}$ & $\begin{array}{r}0.000 \\
0.000\end{array}$ & $\begin{array}{l}518 \\
\Delta \chi_{1}^{2}=\end{array}$ & $\begin{array}{r}0.000 \\
0.000\end{array}$ & $\begin{array}{l}520 \\
\Delta \chi_{1}^{2}\end{array}$ & $\begin{aligned} & 0.000 \\
= & 0.001\end{aligned}$ \\
\hline
\end{tabular}

*The first model in each case allows the twin pair correlations to differ between sexes. Model 2 constrains the twin-pair correlations to be equal for both sexes, and the third model constrains all twin-pair correlations to zero. 
analysis techniques. However, multivariate analysis of the four ordinal variables using Cholesky factorisation methods [Neale and Cardon, 1992] provided some evidence of genetic effects, with additive genetic factors unable to be completely removed from models for mosquito susceptibility at age 12 or 14 .

\section{Comparative Rating Data}

Table V presents the contingency tables for MZ and DZ twins for the comparative rating item pertaining to mosquito bite frequency. In order to determine whether the perceived differences between twins reflect real underlying trait differences [Eaves et al., 1991], twin pairs who agreed on a within-pair difference in mosquito bite frequency were tested for differences in responses to the four ordinal questionnaire items. As demonstrated by Table VI, items relating to being annoyed by mosquitoes, duration of bite itchiness and description of mosquito bites (visits 1 and 2) all exhibited statistically significant differences in responses between twins with "reliable" differences. Response differences were in the expected direction (that is, twins who were rated by themselves and their co-twin as being more affected by mosquitoes also had higher self-report values for the ordinal variables), thus providing internal validation of the comparative measure.

Table VII summarises the parameter estimates and hypothesis testing statistics for a range of comparative rating models for subjects at visit 1 (approximately age 12). Model 1 is a non-scalar sex limitation model, with information on sex differences obtained from the non-central cells on the main diagonal of the contingency table for opposite-sex twins. This analysis was run from a number of different starting values, with identical results. The second model fixed the correlation between opposite-sex twins at 0.5 , but still permitted the mean values for males and females to differ. A significant difference in the means for males and females was demonstrated by model 3, while the estimated threshold parameters (measured as departures from the mean) for males and females may be equated (model 4) without significant worsening of the model fit. Within-pair variances attributable to environmental effects and genetic effects were also not significantly different for males and females (models 5 and 6). However, omission of genetic effects from the model (model 7) is clearly not supported by the data $\left(\chi_{1}^{2}=6.431, P=0.011\right.$ ).

The most parsimonious model, model 6 , estimates the within-pair variance components for genetic and environmental effects to be 1.70 and 0.30 , respectively. Approximately $85 \%$ of these reliable within-pair differences are therefore accounted for by genetic effects. Similar results were obtained for the same suite of comparative ratings models for the data collected when the twin pairs where re-interviewed at age 14 , with the estimate of the proportion of reliable within-family variance due to genetic effects consistent with that obtained at visit $1(82 \%)$.

\section{DISCUSSION}

Test-retest correlations indicated only a moderate consistency of response over the 2-11-week interval for ordinal measures, and were particularly low for the variable relating to being annoyed by mosquitoes. Even poorer correlation between responses was observed over the 2-year interval between visit 1 and visit 2 . The variability with time of individual attractiveness to mosquitoes [Khan et al., 1971], and the possibility of an 
TABLE V. Contingency Tables of Mosquito Bite Frequency Comparative Ratings Given by Australian Adolescent Complete Twin Pairs at Visit 1 (Age 12 Years) and Visit 2 (Age 14 Years)*

\begin{tabular}{|c|c|c|c|c|c|c|c|c|c|c|c|c|c|c|c|}
\hline & \multicolumn{3}{|c|}{ MZF (Visit 1) } & \multicolumn{3}{|c|}{ MZM (Visit 1) } & \multicolumn{3}{|c|}{ DZF (Visit 1) } & \multicolumn{3}{|c|}{ DZM (Visit 1) } & \multicolumn{3}{|c|}{ DZFM (Visit 1) } \\
\hline & $\mathrm{T} 1>\mathrm{T} 2$ & $\mathrm{~T} 1=\mathrm{T} 2$ & $\mathrm{~T} 1<\mathrm{T} 2$ & $\mathrm{~T} 1>\mathrm{T} 2$ & $\mathrm{~T} 1=\mathrm{T} 2$ & $\mathrm{~T} 1<\mathrm{T} 2$ & $\mathrm{~T} 1>\mathrm{T} 2$ & $\mathrm{~T} 1=\mathrm{T} 2$ & $\mathrm{~T} 1<\mathrm{T} 2$ & $\mathrm{~T} 1>\mathrm{T} 2$ & $\mathrm{~T} 1=\mathrm{T} 2$ & $\mathrm{~T} 1<\mathrm{T} 2$ & $\mathrm{~T} 1>\mathrm{T} 2$ & $\mathrm{~T} 1=\mathrm{T} 2$ & $\mathrm{~T} 1<\mathrm{T} 2$ \\
\hline $\mathrm{T} 1>\mathrm{T} 2$ & 7 & 11 & 2 & 10 & 3 & 4 & 14 & 1 & 6 & 10 & 7 & 8 & 25 & 9 & 14 \\
\hline $\mathrm{T} 1=\mathrm{T} 2$ & 4 & 32 & 5 & 1 & 20 & 4 & 3 & 10 & 6 & 1 & 12 & 8 & 11 & 19 & 14 \\
\hline \multirow[t]{3}{*}{$\mathrm{T} 1<\mathrm{T} 2$} & 1 & 1 & 7 & 3 & 8 & 5 & 0 & 4 & 16 & 2 & 4 & 9 & 1 & 4 & 13 \\
\hline & \multicolumn{3}{|c|}{ MZF (Visit 2) } & \multicolumn{3}{|c|}{ MZM (Visit 2) } & \multicolumn{3}{|c|}{ DZF (Visit 2) } & \multicolumn{3}{|c|}{ DZM (Visit 2) } & \multicolumn{3}{|c|}{ DZFM (Visit 2) } \\
\hline & $\mathrm{T} 1>\mathrm{T} 2$ & $\mathrm{~T} 1=\mathrm{T} 2$ & $\mathrm{~T} 1<\mathrm{T} 2$ & $\mathrm{~T} 1>\mathrm{T} 2$ & $\mathrm{~T} 1=\mathrm{T} 2$ & $\mathrm{~T} 1<\mathrm{T} 2$ & $\mathrm{~T} 1>\mathrm{T} 2$ & $\mathrm{~T} 1=\mathrm{T} 2$ & $\mathrm{~T} 1<\mathrm{T} 2$ & $\mathrm{~T} 1>\mathrm{T} 2$ & $\mathrm{~T} 1=\mathrm{T} 2$ & $\mathrm{~T} 1<\mathrm{T} 2$ & $\mathrm{~T} 1>\mathrm{T} 2$ & $\mathrm{~T} 1=\mathrm{T} 2$ & $\mathrm{~T} 1<\mathrm{T} 2$ \\
\hline $\mathrm{T} 1>\mathrm{T} 2$ & 6 & 5 & 1 & 1 & 5 & 1 & 9 & 4 & 1 & 7 & 3 & 1 & 14 & 9 & 1 \\
\hline $\mathrm{T} 1=\mathrm{T} 2$ & 3 & 18 & 4 & 1 & 16 & 5 & 0 & 8 & 5 & 1 & 7 & 1 & 2 & 11 & 6 \\
\hline $\mathrm{T} 1<\mathrm{T} 2$ & 0 & 4 & 6 & 1 & 3 & 2 & 0 & 1 & 11 & 1 & 0 & 7 & 0 & 3 & 6 \\
\hline
\end{tabular}

*Column 1 gives the rating by twin 1, with the corresponding ratings given by twin 2 read across the row. For opposite-sex twins, twin 1 is always the female twin.

"Reliable" comparisons (those on which both twins agree) are highlighted in bold. 
TABLE VI. Fisher's Exact Test (2-Tail) for a Difference in Responses to Ordinal Measures of Mosquito Bite Effect on Australian Adolescent Twin Pairs Who Agreed on a Within-Pair Difference for the Comparative Rating of Mosquito Bite Frequency

\begin{tabular}{lcc}
\hline Variable & Age $=12$ years $\left(\mathrm{N}_{\text {pair }}=116\right)$ & Age $=14$ years $\left(\mathrm{N}_{\text {pair }}=82\right)$ \\
\hline Annoyed by mosquitoes & $P<0.001$ & $P=0.026$ \\
Use mosquito repellent & $P=0.032$ & $P=0.362$ \\
Duration of bite itchiness & $P<0.001$ & $P<0.001$ \\
Bite description & $P<0.001$ & $P=0.032$ \\
\hline
\end{tabular}

individual's reaction to mosquito bites changing with increasing exposure [McKiel and West, 1961] could not be considered as possible causes of the variation in results in the 2-11-week retesting period, although they may have had some effect over the 2-year interval between visit 1 and visit 2 . Familial factors were found to influence the majority of these variables at both visits, with additive genetic effects found to be significant in multivariate analysis of the four ordinal variables.

By contrast, the short-term reliability of the comparative rating variable is considerably higher (0.81, although only based on 48 cases). At both visit 1 and visit 2 , significant genetic effects were detected, with estimates of the proportion of reliable within-family variance due to genetic effects of 85 and $82 \%$, respectively. This estimate is close to the estimate of short-term reliability and suggests that most reliable variance is genetic. Mean values, category response thresholds, and proportions of within-pair variances attributable to environmental and genetic effects were not significantly different for males and females. Twins with "reliable" differences for the comparative rating variable also demonstrated significant differences between their responses to other mosquito-related questionnaire items, indicating that the comparative rating variable is a valid measure of true underlying trait differences [Eaves et al., 1991]. A considerably lower long-term correlation was observed between individual responses at visit 1 and visit 2 (0.67). Since strong genetic effects have been detected at both ages, this suggests that different genes may be responsible for the effects seen in the different age groups, although the size of the effect remains more or less constant. Most of our subjects are going through puberty, a time of rapid physiological change presumably accompanied by large changes in gene expression, between the ages of 12 and 14. It would not be surprising, therefore, that control of a function as complex as mosquito bite susceptibility might also undergo some change in expression.

Unlike questionnaire items used in domains of human experience, the ordinal variables in this study have no widely recognised standard against which personal experience can be compared. Degree of annoyance expressed by individuals, for example, has been found in a community study to be highly variable at a given mosquito trap count, with a lower than expected correlation between degree of annoyance and self-reported bite count [Read et al., 1994]. This may at least partly account for the detection of only weak genetic signals in the ordinal data. Comparative rating items, on the other hand, provide a convenient standard for comparison, in this case the respondent's own twin. At this age in particular, twins would generally be in daily contact and have ample opportunity to compare similarity of experiences. Comparative ratings are hence a potentially valuable tool for complementing and improving the results obtained from more conventional absolute measures.

There are some potential limitations to this study. Firstly, this analysis only 
TABLE VII. Summary of Parameter Estimates and Hypothesis Testing Statistics for Various Comparative Rating

Models of Mosquito Bite Frequency on Australian Adolescents, Visit 1 (Age 12 years) $\left(\mathrm{N}_{\text {pair }}=359\right) *$

\begin{tabular}{|c|c|c|c|c|c|c|c|c|c|c|c|c|c|}
\hline \multirow[b]{2}{*}{ Model } & \multicolumn{8}{|c|}{ Model Parameters } & \multicolumn{5}{|c|}{ Model Fit } \\
\hline & $\mathrm{T}_{\mathrm{m}}$ & $\mathrm{T}_{\mathrm{f}}$ & $\sigma_{e_{m}}^{2}$ & $\sigma_{e f}^{2}$ & $\sigma_{g_{m}}^{2}$ & $\sigma_{g f}^{2}$ & $\overline{\mathrm{M}}-\overline{\mathrm{F}}$ & $r_{\mathrm{aPM}}$ & $\begin{array}{c}-2 \log \\
\text { likelihood }\end{array}$ & df & $\begin{array}{c}\text { Comparison } \\
\text { model }\end{array}$ & $\Delta \chi_{1}^{2}$ & $p_{\text {comparison }}$ \\
\hline 1 & 0.73 & 0.75 & 0.35 & 0.19 & 0.89 & 3.37 & 0.63 & 1.00 & 736.309 & 32 & - & - & 一 \\
\hline 2 & 0.73 & 0.75 & 0.35 & 0.19 & 0.89 & 3.37 & 0.63 & $1.00^{\mathrm{a}}$ & 736.309 & 33 & 1 & 0.000 & 1.000 \\
\hline 3 & 0.70 & 0.74 & 0.29 & 0.18 & 0.85 & 3.17 & $0.00^{\mathrm{a}}$ & $1.00^{\mathrm{a}}$ & 745.347 & 34 & 2 & 9.038 & 0.003 \\
\hline 4 & $0.74^{\mathrm{b}}$ & $0.74^{\mathrm{b}}$ & 0.36 & 0.18 & 0.90 & 3.34 & 0.63 & $1.00^{\mathrm{a}}$ & 736.337 & 34 & 2 & 0.028 & 0.867 \\
\hline 5 & $0.73^{\mathrm{b}}$ & $0.73^{\mathrm{b}}$ & $0.31^{\mathrm{c}}$ & $0.31^{\mathrm{c}}$ & 0.95 & 2.25 & 0.60 & $1.00^{\mathrm{a}}$ & 727.248 & 35 & 4 & 0.911 & 0.340 \\
\hline 6 & $0.74^{b}$ & $0.74^{\mathrm{b}}$ & $0.30^{c}$ & $0.30^{c}$ & $1.70^{d}$ & $1.70^{d}$ & 0.62 & $1.00^{\mathrm{a}}$ & 739.004 & 36 & 5 & 1.756 & 0.185 \\
\hline 7 & $0.74^{\mathrm{b}}$ & $0.74^{\mathrm{b}}$ & $0.89^{c}$ & $0.89^{c}$ & $0.00^{\mathrm{a}}$ & $0.00^{\mathrm{a}}$ & 0.58 & $1.00^{\mathrm{a}}$ & 745.435 & 37 & 6 & 6.431 & 0.011 \\
\hline
\end{tabular}

$* \mathrm{~T}_{\mathrm{m}}=$ threshold parameter for males; $\mathrm{T}_{\mathrm{f}}=$ threshold parameter for females; $\sigma_{g_{m}}^{2}=$ within-pair variance attributable to genetic effects, males; $\sigma_{g f}^{2}=$ within-pair variance attribute to genetic effects, females; $\overline{\mathrm{M}}-\overline{\mathrm{F}}=$ difference in means between sexes; $r_{\mathrm{a} F M}=$ correlation between genes affecting trait in opposite sex twin pairs; $\sigma_{e_{m}}^{2}=$ within-pair variance attributable to environmental effects, males; $\sigma_{e f}^{2}=$ within-pair variance attributable to environmental effects, females.

${ }^{\text {a }}$ Parameters fixed in given model.

b,c,d Parameters specified as equal in given model. 
considers adolescents, and the results may not be valid for other age groups, particularly given that age has been found to be a factor in the attraction of mosquitoes to potential hosts [Maibach et al., 1966]. Secondly, the validity of the comparative rating method is reliant on the accuracy of assumptions regarding the normality of the underlying distribution of the latent trait. Thirdly, at least several factors are likely to contribute to the perception of being bitten, two of which are the actual frequency of being bitten and the severity of the reaction to the mosquito bite. Since the twins in this study will have been exposed to a range of mosquito species with different specific attractants, it seems likely that if the genetic effects on the perception of being bitten by mosquitoes is mediated through differences in biting rates of mosquitoes, then this may be mediated, in turn, through modulation of more attractive signals such as $\mathrm{CO}_{2}$, moisture, and temperature plumes.

\section{ACKNOWLEDGMENTS}

The authors thank Ann Eldridge and Marlene Grace for data collection, John Pearson for data management, Adam Storey and Bradley Christiansen for early contributions to data analysis, and Dr. Kim-Anh Do for helpful discussions.

\section{REFERENCES}

Aitken JF, Green A, Eldridge A, Green L, Pfitzner J, Battistutta D, Martin NG. 1994. Comparability of naevus counts between and within examiners, and comparison with computer image analysis. Br J Cancer 69:487-91.

Brouwer R. 1960. Variations in human body odour as a cause of individual differences of attraction for malaria mosquitoes. Trop Geogr Med 2:186-92.

Brown AW. 1966. The attraction of mosquitoes to hosts. JAMA 196:249-52.

Eaves LJ, Neale MC, Meyer JM. 1991. A model for comparative ratings in studies of within-family differences. Behav Genet 21:531-6.

Fradin MS. 1998. Mosquitoes and mosquito repellents: a clinician's guide. Ann Int Med 128:931-40.

Gillies MT. 1980. The role of carbon dioxide in host-finding by mosquitoes (Diptera: Culicidae): a review. Bull Ent Res 70:525-32.

Jöreskog KG, Sörbom D. 1998. LISREL 8.20 and PRELIS 2.20 [computer program]. Chicago: Scientific Software Inc.

Khan AA, Maibach HI, Strauss WG. 1971. A quantitative study of variation in mosquito response and host attractiveness. J Med Entomol 8:41-3.

Knols BGJ, De Jong R. 1996. Limburger cheese as an attractant for the malaria mosquito Anopheles gambiae s.s. Parasitol Today 12:159-61.

Maibach HI, Khan AA, Strauss WG. 1966. Attraction of humans of different age groups to mosquitoes. J Econ Entomol 59:1302-3.

McKiel JA, West AS. 1961. Nature and causation of insect bite reactions. Pediatr Clin North Am 8:795-815.

Mellanby K. 1946. Man's reaction to mosquito bites. Nature 158:554.

Neale MC. 1997. Mx: statistical modeling, 4th ed. Richmond, VA: Department of Psychiatry, Medical College of Virginia.

Neale MC, Cardon LR. 1992. Methodology for genetic studies of twins and families. Dortrecht: Kluwer Academic Publishers.

Read NR, Rooker JR, Gathman JP. 1994. Public perception of mosquito annoyance measured by a survey and simultaneous mosquito sampling. J Am Mosq Control Assoc 10:79-87.

Ritchie SA, Fanning ID, Phillips DA, Standfast HA, McGinn D, Kay BH. 1997. Ross River Virus in mosquitoes (Diptera: Culicidae) during the 1994 epidemic around Brisbane, Australia. J Med Entom 34:156-9. 
SAS Institute. 1996. SAS Version 6.11 [computer program]. Cary, NC: SAS Institute.

Snow WF. 1970. The effect of a reduction in expired carbon dioxide on the attractiveness of human subjects to mosquitoes. Bull Ent Res 60:43-8.

Wright RH. 1975. Why mosquito repellents repel. Sci Am 233:104-11. 